\title{
Radio Frequency Fingerprinting Driven Drone Identification Based on Complex-valued CNN
}

\author{
Hao Gu${ }^{1}$, Yun Lin², Yu Wang ${ }^{1}$, Hao Huang ${ }^{1}$, Sheng Hong ${ }^{1}$, Jie Yang ${ }^{1}$, \\ Miao Liu ${ }^{1}$, Jinlong Sun $^{1}$, and Guan Gui ${ }^{1 *}$ \\ 1. College of Telecommunication and Information Engineering, Nanjing University of Posts and \\ Telecommunications, Nanjing, 210003 China \\ 2. College of Information and Communication Engineering, Harbin Engineering University, Harbin, 150001, \\ China \\ E-mails: \{1218012510, guiguan\}@njupt.edu.cn
}

\begin{abstract}
Drone detection and identification technique is of great significance both in the military and civilian fields. Radio frequency (RF) fingerprinting of drone is considered as one of promising techniques due to its uniqueness. Deep learning based RF fingerprinting identification technique can extract hidden features in RF data and then achieve excellent performance. Motivated by this idea, this paper proposes a drone identification method using complex-valued convolutional neural network (CNN) algorithm with higher classification accuracy and faster equipment running time. The complex-valued CNN method convolves the complex convolutional kernel and the real and imaginary parts of the data features separately. In order to verify the proposed method, five state-of-the-art recognition algorithms are adopted to compare their recognition performance and equipment efficiency. Simulation results show that our proposed drone identification method can efficiently recognize the signal of various drones within less computation time.
\end{abstract}

Keywords: Drone identification, complex-valued CNN, intelligent recognition, RF fingerprinting, deep learning.

\section{Introduction}

In recent years, drones have quietly infiltrated into people's daily lives and have brought drastic changes to people's lives [1]. In rural areas, drones have greatly improved transportation capacity in remote areas and agricultural production efficiency. In cities, drones not only play an important role in solving the problem of logistics, but also provide detailed land use information and law enforcement evidence for urban planning, construction and management, such as road construction, traffic patrols and city law enforcement. However, the widespread application of drones is bound therefore raising a series of technical and public safety issues [2]. Relevant regulators must be able to adopt effective technologies to detect and identify various drones [3].

RF fingerprinting can be used to detect and identify drones. Due to the different locations and configuration parameters of each smart device, the signal sent by smart devices all has their specific fingerprint [4]. The RF fingerprinting identification technology collects signals from various devices 
with their own fingerprint through the receiver and distinguishes them. Specifically, RF fingerprintingbased identification method usually includes two parts: training and identification [5]. The training part means that after receiving devices such as antennas collect wireless signals from various smart devices, they collect their inherent signal characteristics (fingerprint). The identification part means that when an unknown signal is received, we can complete the identification of the unknown signal according to the characteristics of signal that have been collected above [6]. Generally, the above-mentioned RF fingerprinting identification process does not require complicated calculations and can be directly embedded in the host of the receiver. This technology is very suitable for various types of internet of things (IoT) devices [7].

Additionally, deep learning algorithms are widely used in wireless communications [8][9][10] and achieve good performance in physical layer [11][12]. Y. Wang et al. [13] used two layers of convolutional neural network $(\mathrm{CNN})$ networks to identify the modulation signals under unknown channels. What's more, RF fingerprinting technology also uses deep learning algorithms. J. Yu et al. [14] proposed a multisampling convolutional neural network (MSCNN) to identify ZigBee devices efficiently with low cost. Therefore, the existing technologies RF fingerprinting recognition technologies based on deep learning can also be applied to drone detection and recognition methods.

Based on the RF data collected from true drone devices, this paper proposes a drone identification method via complex-valued CNN in order to identify genuine drone devices. Two RF receivers are used to receive the high-frequency and low-frequency parts of the drone RF data, which are $2.4 \mathrm{GHz}$ wifi signals. Then discrete fourier transform (DFT) is performed on the RF data from the two receivers and they are connected together to form the entire RF spectrum of drones. After processing the drone signal, we convolve the complex convolutional kernel and the real and imaginary parts of the data features separately and compare the performance of five different algorithms who are trained on independent datasets. Simulation results are given to confirm equipment running time and identification performance of our proposed method.

The remainder of this paper is organized as follows. In Section 2, we introduce the drone identification system and our specific datasets. Section 3 presents the analysis of two drone identification methods and their neural network structure. Section 4 introduces the simulation results and we make conclusion in section 5 .

\section{System Model and Datasets}

\subsection{System model}

In our proposed drone identification system, we use complex-valued CNN algorithm to detect and recognize the signals of different drones. This system is shown in Fig.1, which consists of three parts: signal processing part, classification part and evaluation part. The first part is to receive and pre-process the complex RF data of drones. Further, the second part is to train the complex-valued CNN algorithm based on those RF signals in order to classify unknown received drone signals. Last but not least, we could analyze the identification accuracy and other evaluation criteria based on the output results of our proposed drone identification system in the evaluation part.

\subsection{RF data based drone datasets}

Based on the system model, drone datasets are composed of the complex RF data collected from real drones. These real drone signals are provided by a large open source drone database. This database has 
collected many very valuable real drone RF data through the following three modules: drones under analysis module, fight control module and RF sensing module. What's more, we used four types of drone signals in this database, as is shown in Fig. 1. We can see from the figure that the drone signal datasets contain one type of Background activities (collecting RF background activities data when drones are off) and three types of drone activities (collecting RF background activities data when drones are on). More importantly, three types of drone activities represent three different brands of drones, which means different brands have different prices, protocols, and technologies. After IQ sampling the RF data of each drone activity, we can get drone signal datasets.

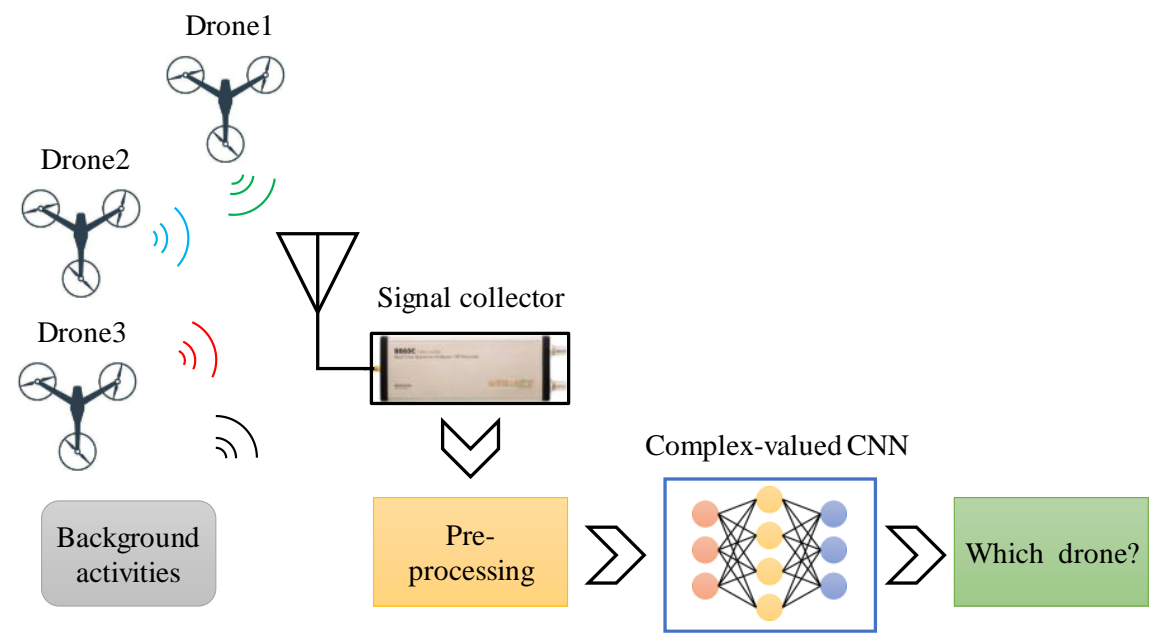

Fig. 1. System model of our proposed drone identification method.

Additionally, we used a total of 4,400 drone signal samples in our experiment, with an average of 1100 signal samples per drone activity. These samples are randomly assigned to a proportion of 7: 3 for the training and testing of the Complex-valued CNN. The length of each sample is 2048. What's more, in order to better extract the features in the datasets, we split each sample into in-phase and quadrature component, whose data form is a real-valued matrix of $2 \times 2048$. These datasets will be used as the input to the drone identification via complex-valued CNN system so that it can identify different drone signals. Last but not least, Table 1 shows the software and hardware configuration used in our data preprocessing, neural networks training and algorithm model verification.

Table 1. Software and hardware configuration details.

\begin{tabular}{ll}
\hline Running environment & Details \\
\hline Software & Spyder, MATLABR2019a \\
Language & Python3.7.1 \\
Software library & Keras 2.2.2 \\
CPUs & 8 Intel Xeon E3 (x86_64) \\
GPUs & 4 NVIDIA GTX1080Ti \\
Operating system & Ubuntu 16.04.1-Linux \\
\hline
\end{tabular}




\section{Drone Identification Methods}

In this section, two different drone identification methods based on deep learning is introduced. One of them is traditional drone detection and identification method based on fully connected deep neural network (FCN), the another one is drone identification via complex-valued CNN. Each layer of neural network and specific parameters of them will be described in details.

\subsection{Traditional drone detection and identification method based on FCN}

The architecture of traditional drone detection and identification method based on FCN can be divided into three parts: input layer, hidden layer and output layer. Obviously, we need to focus on the hidden layer who contains 4 fully connected (FC) layers. The number of neurons in the first, second, and third FC layers is 256,128 , and 64, respectively. After every fully connected layer, batch normalization $(\mathrm{BN})$ layer and activation function layer that uses ReLU function is added. Moreover, adding the Dropout layer after each layer is also essential, who can reduce network parameters and avoid overfitting. The number of neurons in the fourth FC layer is the same as the number of drone signals we want to identify. In addition, by comparing the true labels of the input layer with the predicted labels of the output layer, we can know the recognition performance of this traditional FCN method.

\subsection{Drone identification via complex-valued CNN}

Our proposed drone identification method via complex-valued CNN is introduced in this section. Complex-valued $\mathrm{CNN}$ is an extension of the traditional real-value $\mathrm{CNN}$ in the complex field. Since the drone signal data comes in the form of IQ data, we have reasons to believe that complex-valued CNN will have a stronger ability to extract features. Hence, we add real-valued CNN models in our simulation experiments in order to compare the identification performance of drone signals with complex-valued CNNs.

As we all know, convolutional operation has a very important position in CNN, which can sparse network parameters and extract data features within all directions. Therefore, the significance of complex convolutional operation is self-evident in complex-valued CNN, and it can be achieved by multiple realvalued convolutional operations. First, we introduce a complex vector $\mathbf{h}$ and a complex filter matrix $\mathbf{W}$, whose definition is as follows:

$$
\begin{gathered}
h=x+i y \\
W=A+i B
\end{gathered}
$$

where $\mathbf{x}, \mathbf{y}$ are real vectors, and $\mathbf{A}, \mathbf{B}$ are real matrices. The convolution process of $\mathbf{h}$ and $\mathbf{W}$ is as follows:

$$
W * h=(A * x-B * y)+i(B * x+A * y)
$$

from this formula we can see that the complex convolutional operation can be split into the sum of the convolution of the real and imaginary parts of one vector $\mathbf{h}$ and the same two parts of another matrix $\mathbf{W}$, respectively. According to this formula, we can infer the complex convolution operation between the complex feature map $M$ and the complex convolution kernel $K$ :

$$
M * K=\left(M_{R} K_{R}-M_{I} K_{I}\right)+i\left(M_{R} K_{I}+M_{I} K_{R}\right)
$$

where the subscript $R$ represents the real part and the subscript $I$ represents the imaginary part. 
After introducing the compress convolutional operation, the structure of complex-valued CNN in our proposed drone identification system is shown in Fig. 2. It consists of two parts: complex convolutional layer and complex FC layer. On the one hand, the first complex convolutional layer contains 128 filters whose convolutional kernel is 16 and the second complex convolutional layer is composed of 64 filters, whose convolutional layer is 8. After the complex convolutional layers, there are also three complex FC layers. The number of neurons in the first, second and third complex FC layer is 256, 128 and 2 respectively. In order to speed up the training speed of the deep network and avoid overfitting, we add a complex BN layer and a Dropout layer after each complex convolution layer or FC layer. Specifically, the number of neurons in the third complex FC layer is determined by the number of drone activities that we want to identify, and the activation function of this layer is Softmax, which can output the predicted probability of each category.

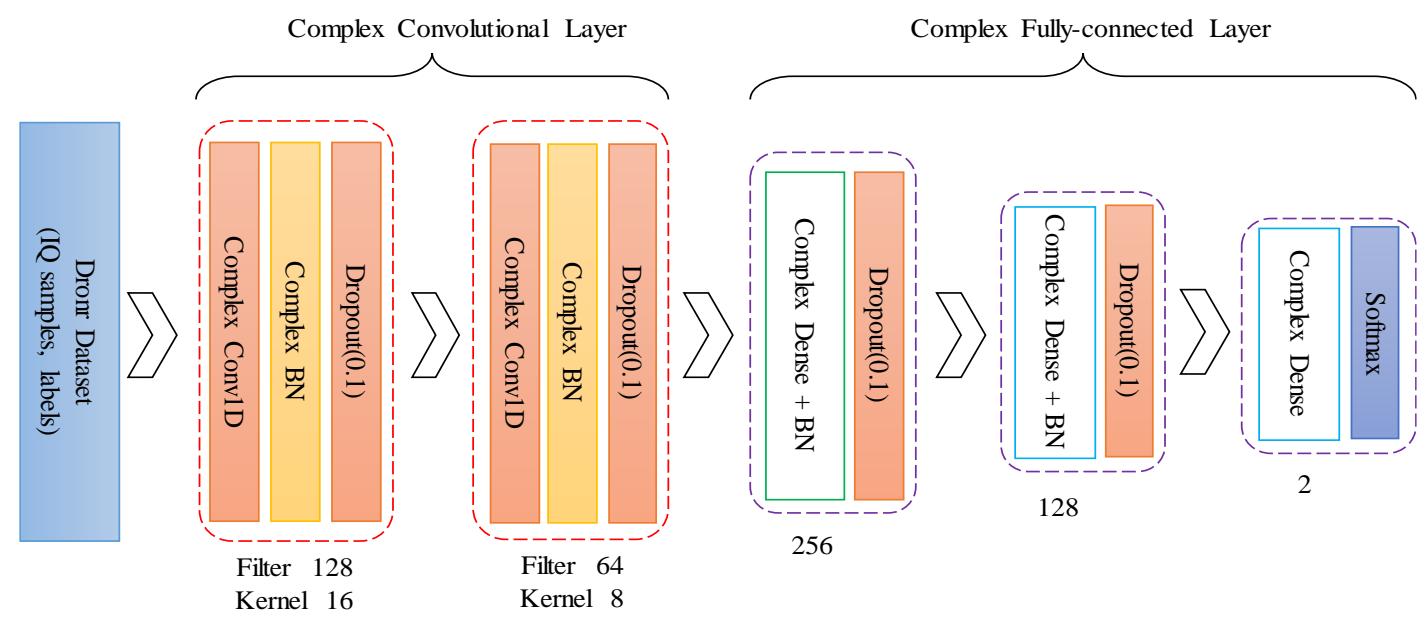

Fig. 2. The structure of complex-valued $\mathrm{CNN}$ in our proposed drone identification system.

\section{Simulation results}

In this section, we analyze the classification accuracy of five drone identification methods for our drone signal datasets contain one type of background activities and three types of drone activities. These algorithm models are trained on independent datasets, which $70 \%$ of them are used for training and $30 \%$ of them are used for testing. We comprehensively evaluate the complex-valued CNN-based algorithm proposed in this paper in terms of model classification accuracy, prediction time of a single sample, and confusion matrix.

\subsection{Classification performance of drone identification model}

Table 2 shows the classification accuracy and GPT-Time of single sample in different drone identification models. These five methods including complex-valued CNN (our proposed), real-valued CNN, traditional drone identification method based on FCN, long short-term memory (LSTM), decision tree (DT) and support vector machine (SVM). First, we can clearly see from the table that deep learning methods get higher recognition accuracy than machine learning algorithms (DT, SVM). The traditional FCN algorithm is $30 \%$ higher than LSTM algorithm and reaches $85 \%$. Additionally, two CNN-based recognition algorithms both get accuracy of more than $90 \%$. Our proposed complex-valued CNN-based 
identification algorithm has the highest accuracy rate, even reaching $99.5 \%$. Because of lacking the ability to extract features in the complex domain, real-valued CNN algorithm's accuracy rate is $7 \%$ lower.

Next, we focus on the running time of a single sample within all algorithms, who are all running on the same machine. We can see from the third column of the table that the running GPU-time of SVM algorithm is the longest, reaching $63 \mathrm{~ms}$, who is dozen times of other algorithms. The reason is that SVM algorithm is not suitable for classification models with too long data length. The running GPU-time of DT is the shortest, only $0.068 \mathrm{~ms}$. However, the GPU-time of neural networks is basically similar. Complex-valued $\mathrm{CNN}$ algorithm runs slightly efficient than other deep learning algorithms because it has fewer neurons. Taken together, complex-valued CNN algorithm not only has higher classification accuracy, but also has less running GPU-time.

Table 2. Accuracy and GPU-Time of each drone identification model.

\begin{tabular}{ccc}
\hline Algorithms & $\begin{array}{c}\text { Accuracy } \\
(\%)\end{array}$ & $\begin{array}{c}\text { GPU-Time } \\
\text { (ms/sample) }\end{array}$ \\
\hline Complex-valued CNN (proposed) & $\mathbf{9 9 . 5 0}$ & $\mathbf{1 . 3 4 4 8}$ \\
Real-valued CNN & 92.93 & 5.0233 \\
FCN & 85.05 & 1.6006 \\
LSTM & 55.63 & 2.0082 \\
DT & 53.93 & 0.0685 \\
SVM & 23.48 & 63.5852 \\
\hline
\end{tabular}

\subsection{Confusion matrices of deep learning algorithm models}

Finally, we provide four confusion matrix of deep learning algorithm models in Fig. 3, which facilitate some details of this four algorithm performance. As is shown in this figure, BG represents the background activities label and D1 D3 represent the drone1 drone3 activities label. By observing the squares on the diagonal of all confusion matrices, who represent correctly predicted labels, we can distinguish the two CNN-based algorithms from other deep learning algorithms. LSTM's confusion matrix is very confusing, and it is almost impossible to accurately predict all drone activities signals. FCN algorithm just performs well in D3 labels. In contrast, two CNN algorithms perform well in predicting three types of drone activities, and the difference in the accuracy of predicting background activities signals has led to their overall gap. The above shows that the complex-valued CNN can accurately distinguish every drone signals so that our proposed method has better recognition ability and robustness. 


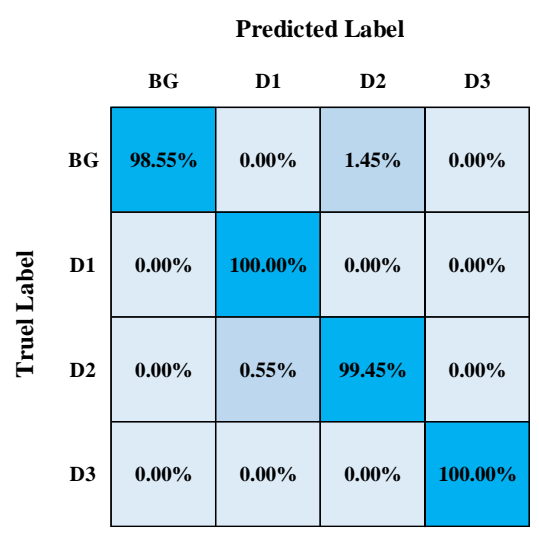

(a) Complex-valued CNN (proposed)

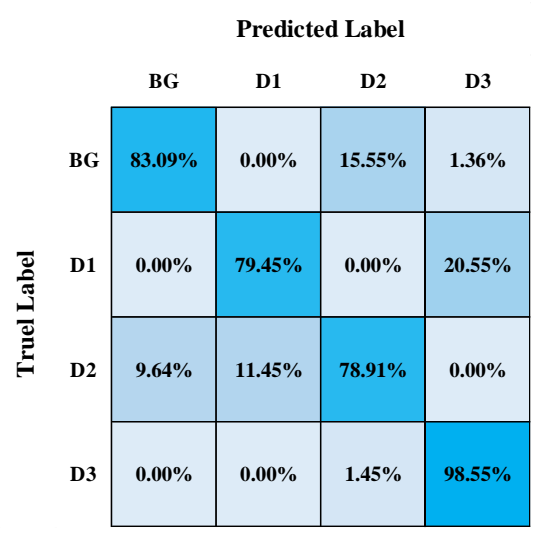

(c) FCN

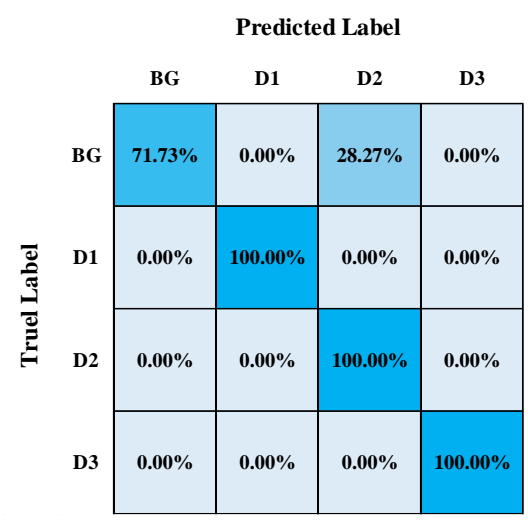

(b) Real-valued CNN

Predicted Label

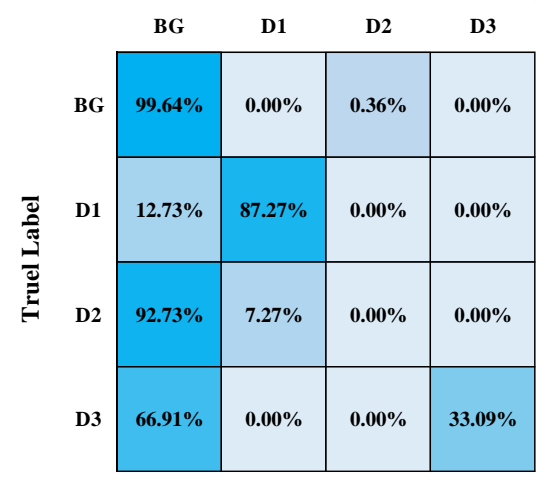

(d) LSTM

Fig. 3. Confusion matrices of deep learning algorithm models.

\section{Conclusions}

In this paper, we have proposed a drone identification method via complex-valued CNN driven by RF fingerprinting. The proposed complex-valued CNN algorithm is based on real-valued CNN by convolve the real and imaginary parts of the convolution kernel separately. Then, we compare complexvalued $\mathrm{CNN}$ with other popular algorithms by training and verifying the classification accuracy and recording running time of them. Simulation results show that the proposed drone identification method via complex-valued CNN achieve excellent performance in identify drone signals and need less equipment loss. In the future work, we will consider expanding the drone datasets and trying to identify $\mathrm{RF}$ data of different running modes in the same drone. More importantly, we expect to prune some neurons in the complex-valued CNN proposed in this paper in order to ensure the identification accuracy and improve the network operation efficiency.

\section{References}

[1] S. Al-Emadi, A. Al-Ali, A. Mohammad, and A. Al-Ali, "Audio based drone detection and identification using deep learning," International Wireless Communications and Mobile Computing Conference (IWCMC), Tangier, Morocco, June 24-28, 2019, pp. 459-464.

[2] H. M. Oh, H. Lee, and M. Y. Kim, "Comparing Convolutional Neural Network(CNN) models for machine learning-based drone and bird classification of anti-drone system," Int. Conf. Control. Autom. Syst. (ICCAS), 
Jeju, South Korea, Oct. 15-18 2019, pp. 87-90.

[3] A. Shoufan, H. M. Al-Angari, M. F. A. Sheikh, and E. Damiani, "Drone pilot identification by classifying radiocontrol signals," IEEE Trans. Inf. Forensics Secur., vol. 13, no. 10, pp. 2439-2447, 2018.

[4] L. Peng, A. Hu, J. Zhang, Y. Jiang, J. Yu, and Y. Yan, "Design of a hybrid RF fingerprint extraction and device classification scheme," IEEE Internet Things J., vol. 6, no. 1, pp. 349-360, 2019.

[5] Y. Lin, X. Zhu, Z. Zheng, Z. Dou, and R. Zhou, "The individual identification method of wireless device based on dimensionality reduction and machine learning,” J. Supercomput., vol. 75, no. 6, pp. 3010-3027, 2019.

[6] K. Yang, J. Kang, J. Jang, and H. N. Lee, "Multimodal sparse representation-based classification scheme for RF fingerprinting,” IEEE Commun. Lett., vol. 23, no. 5, pp. 867-870, 2019.

[7] K. Satyanarayana, M. El-Hajjar, A. A. M. Mourad, and L. Hanzo, "Deep learning aided fingerprint-based beam alignment for mmWave vehicular communication," IEEE Trans. Veh. Technol., vol. 68, no. 11, pp. 10858$10871,2019$.

[8] H. Huang et al., "Deep learning for physical-layer 5G wireless techniques: Opportunities, challenges and solutions," IEEE Wirel. Commun. Mag., vol. 27, no. 1, pp. 214-222, 2019.

[9] H. Huang, Y. Peng, J. Yang, W. Xia, and G. Gui, "Fast beamforming design via deep learning," IEEE Trans. Veh. Technol. Technol., vol. 69, no. 9, pp. 1065-1069, 2019.

[10]G. Gui, H. Huang, Y. Song, and H. Sari, “Deep learning for an effective non-orthogonal multiple access scheme,” IEEE Trans. Veh. Technol., vol. 67, no. 9, pp. 8440-8450, 2018.

[11] Y. Wang, J. Yang, M. Liu, and G. Gui, "LightAMC: Lightweight automatic modulation classification using deep learning and compressive sensing," IEEE Trans. Vechicular Technol., vol. 69. no. 3, pp. 3491-3495, 2020.

[12] H. Gu, Y. Wang, S. Hong, and G. Gui, "Blind channel identification aided generalized automatic modulation recognition based on deep learning,” IEEE Access, vol. 7, pp. 110722-110729, 2019.

[13] Y. Wang, M. Liu, J. Yang, and G. Gui, "Data-driven deep learning for automatic modulation recognition in cognitive radios," IEEE Trans. Veh. Technol., vol. 68, no. 4, pp. 4074-4077, 2019.

[14] J. Yu, A. Hu, G. Li, and L. Peng, “A robust RF fingerprinting approach using multisampling convolutional neural network," IEEE Internet Things J., vol. 6, no. 4, pp. 6786-6799, 2019. 